\title{
Promoting Medication Adherence to Asthma
}

\author{
Weerapong Lilitwat ${ }^{1}$, Yuttiwat Vorakunthada ${ }^{2}$ \\ ${ }^{1}$ Department of Pediatric Critical Care Medicine, University of Iowa Hospitals and Clinics, Iowa City, IA, USA \\ ${ }^{2}$ Department of Internal Medicine, Texas Tech University Health Sciences Center, Lubbock, TX, USA \\ Email: y.vorakunthada@ttuhsc.edu
}

How to cite this paper: Lilitwat, W. and Vorakunthada, Y. (2018) Promoting Medication Adherence to Asthma. Health, 10, 13-19.

https://doi.org/10.4236/health.2018.101002

Received: November 16, 2017

Accepted: January 1, 2018

Published: January 4, 2018

Copyright $\odot 2018$ by authors and Scientific Research Publishing Inc. This work is licensed under the Creative Commons Attribution International License (CC BY 4.0).

http://creativecommons.org/licenses/by/4.0/

\section{(c) (i) Open Access}

\begin{abstract}
Promoting adherence to asthma treatment is an essential aspect of clinical practice. Approximately $60 \%$ of asthmatic patients are non-adherent to asthma regimen, resulting in adverse outcomes and higher costs of care. Non-adherence could be intentional (perceptions of asthma severity, self-manage therapy, fear of side-effects) or non-intentional (forgetful, cost, and misunderstandings). Adherence can be evaluated by patient's reporting, dose counter, electronic metered dose inhaler but using pharmacy records is a more cost-effective method. The most successful strategies to improve patients' adherence is to utilize the principle of patient-centered collaborative care and effective communication. Keys of communication skills consist of establishing a relationship, listening, collaborating on the treatment plan, time management, and implementing effective follow-up interventions. Interventions to improve adherence include providing reinforcement for patients' efforts to change, providing feedback on progress, tailoring education to patients' needs and circumstances and follow-up. Evaluation of health literacy is mandatory for prioritizing information from most to least critical, speaking slowly, avoiding medical jargon, and spending minimal extra time during each visit. Communication technology including texting or interactive voice response is another new strategy that can increase adherence.
\end{abstract}

\section{Keywords}

Adherence, Asthma, Education, Communication, Health Literacy

\section{Introduction}

An estimated 300 million people have asthma worldwide. The Global Initiative for Asthma (GINA) recommends initial regular controller treatment plus as needed short-acting beta agonist (SABA) for medication therapy. However, less than $60 \%$ of asthmatic patients have been reported to use medication regularly 
[1]. This non-adherence burdens both the healthcare costs and undesirable clinical outcomes. The cost of asthma in the industrialized countries such as the USA was reported to be as high as $1 \%-2 \%$ of total healthcare expenditure [2], not including indirect costs such as lost productivity. The impact of non-adherence on asthma is significant, including frequent asthma exacerbation, increased frequency of emergency visits, and hospitalization. This review article focuses on medication non-adherence and relevant strategies to promote adherence.

\section{Medication Non-Adherence}

Despite the advancement of bronchodilators and inhaled corticosteroids, a significant portion of patients continues to experience poor asthma control. One hypothesis is the presence of a phenotype that corresponds to airway inflammation resistant to medications [3]. This group of patients will require repeated, and prolonged oral corticosteroid (OCS) treatment and this predisposed them to a higher risk of medication side effects. Zazzali et al. demonstrated the use of OCS for greater than 30 days per year is associated with higher risk of corticosteroid-related adverse effects [4]. However, the most significant challenges adherence includes inadequate or inappropriate inhaler technique as well as accessibility to medications. One study showed that patient's confidence on correct inhaler usage was low [5].

Evaluating non-adherence can be challenging in clinical practice because there is no standardized method. One study showed that patients frequently over-report their degree of adherence [3]. There are various techniques for adherence monitoring, include dose counters, prescription refill record and electronic monitoring devices to detect adherence; however, cost-effective needs to be further studied.

In general, non-adherence can be divided into two categories: non-intentional and intentional (Table 1). Forgetfulness, cost, and misunderstandings are instances of non-intentional. However, the majority of non-adherence is intended, as patients make conscious decisions based on perceptions of the burden of illness and medical therapy. Furthermore, it could be fear of side-effects, cultural

Table 1. Non-adherence, modified from GINA guideline 2017.

\begin{tabular}{ll}
\hline Non-intentional factor & Intentional factor \\
Forgetfulness & Perceptions of asthma severity \\
Cost & Understanding of self-manage therapy \\
Misunderstandings & Fear of side-effects \\
& Cultural issues \\
& Patient-doctor communication \\
& Understanding of inhaler use \\
\hline
\end{tabular}


issues, lack of understanding of self-manage treatment, and effective patient-doctor communication.

Age may also play a role of non-adherence. In the pediatric population, child knowledge of asthma has been associated with medication adherence [4], but older children are less adherent than their younger counterparts [5]. From these results, children should have some understandings related to disease and management strategies. In adolescents, adherence is even lower due to questioning of the asthma diagnosis, poor understanding of the nature of asthma, perceiving it as an intermittent rather than a chronic condition, medications taken on as-required basis rather than regularly, and not prioritizing asthma treatment in a busy schedule [6] [7] [8]. Educating parents regarding allergy and asthma can also improve adherence to inhaler therapy. Interesting, one study showed that skin prick test in asthma children at emergency department increased adherence to continuity clinic [9].

Mode of delivery and dosing frequency may influence patient adherence to therapy as well. Simpler treatments are often suggestive of improved adherence; however, the evidence for this recommendation is limited [10] [11] [12]. Unsurprisingly, a once-daily oral tablet such as montelukast is more adherent to inhaled corticosteroid [13] [14] [15], but the adherence rate was not different in children [16]. This result might imply the importance of parental factor, which may play a role in adherence in children.

Psychological aspects of asthma including stress, anxiety, and depression may contribute to non-adherence to the medical regimen [17]. Also, family dysfunction and parental criticism have been associated with lower adherence to controller medications [18]. In adults, the best predictor of medication adherence was the presence of a significant other [19]. Similarly, anxiety seems to be associated with bronchospasm in couples with asthma, and medication adherence involves problem-solving [20]. A reasonable hypothesis for future research is that better medication adherence is associated with better problem-solving abilities [17].

\section{Strategies to Improve Adherence}

Patient-centered collaborative care and effective communication have been introduced to improve patients' adherence. This method reinforces that patients are experts of their life and health providers act as an information provider, tailoring information that patients want to know about their health and feedback. This shared decision-making consists of negotiation between patients and clinicians to accommodate patient goals, and preferences significantly improve adherence to asthma pharmacotherapy and clinical outcomes as shown from Wilson et al. study [21]. The results from this study also improved asthma quality of life, lower use of rescue medication, better asthma control, and lung function. Motivational interviewing; another approach, can be briefly integrated into patient encounters to enhance motivation to change among patients not ready to 
change [22]. This technique helps to clarify patients' ambivalence about behavior change and build their intrinsic motivation before being provided education.

Difficulties in communication could be from many barriers [23], including doctor-patient relationship, discouragement of collaboration, resistance by patients, literacy [24], and language barrier [25]. However, communication skills between 2 parties can be improved using these five strategies: establish a relationship, listening, collaborate on the treatment plan, time management, and implement effective follow-up interventions, handling phone calls or text messaging (Table 2) [26]. This study demonstrated significant improvement in patients' adherence to their asthma medication. A step-wise approach is essential to ensure that patients truly understand their medical condition and allow for clarification of any questions that arise.

This will require an ongoing patient-doctor relationship as well as engagement in shared decision-making (SDM). Studies have shown that better communication and SDM improve adherence and thus, improved asthma outcome [21] [27]. This involves identification of non-adherence and underlying causes; follow with an emphasis on the value of the treatment regimen, as well as clarification on patient's perception of the current treatment. Additionally, providing simple and clear instructions, simplifying regimen, and provide customization base on patient's preference have been shown to be beneficial (Table 3).

Research revealed an inadequate emphasis on asthma self-management education as recommended by The National Asthma Education and Prevention Program. Approximately one-third of asthma patient does not receive an asthma action pain (ACT) and training on peak flow measurement, and signs and symptoms of asthma [28]. About 50\% do not receive education on environmental control measures [28]. Increase emphasis on education will inevitably improve adherence.

Potential barriers to asthma control include the patient's health literacy and the patient's perception of their asthma control. Studies show that health literacy is a strong predictor of a person's health, more so than age, income, employment

Table 2. Communication skills to improve adherence.

\footnotetext{
1. Establish a relationship

2. Listening

3. Collaborate on the treatment plan

4. Time management

5. Implement effective follow-up interventions
}

Table 3. Key components of successful adherence promotion intervention.

\footnotetext{
1. Reinforcing patients' efforts to change

2. Providing feedback on progress

3. Tailoring education to patients' needs and circumstances

4. Continuity of care and education
} 
status, education level, and race [29]. Health care providers need to proactively evaluate the patient's ability to read, understand, and act on health care information. Techniques for health literacy improvement include prioritizing information from most to least critical, speaking slowly, avoiding medical jargon, and spending minimal extra time during each visit.

Cultural and social factors are often neglected, especially in patients from non-Western ethnicity. Many ethnic groups have their own health belief and traditional medications. Western medicine is usually utilized during the acute phase of treatment, whereas traditional medicine is used after the acute event is over. The language barrier can also play a role in non-compliance. Again, this requires adequate understanding and education of the chronic nature of asthma. Social factors are essential especially in the pediatric population, as treatment involves the cooperation of the whole family. Poor family support has linked to non-compliance, and this can be prevented through proper backing from healthcare workers.

Providing feedback and monitoring is a powerful behavioral strategy that has shown improved adherence in electronic metered dose inhaler (MDI) or electronic pharmacy record. However, using pharmacy records to monitor and provide feedback is a more cost-effective strategy to improve adherence. In contrast to electronic MDI which is not practical and expensive [30].

Communication technology such as interactive voice response (IVR) is a new strategy to help increase patient motivation and increase patients' ability to manage asthma. A study in children was found that IVR reminder and educational calls improved adherence with steroid inhaler that was approximately one-third higher than a control group [31]. Text messaging is another simple technology that can apply to improve patient adherence.

\section{Conclusion}

Medication non-adherence in asthma patients is a challenge in clinical practice. Patient-centered collaborative care and effective communication skills are the foundation for promoting adherence as demonstrated by clinical evidence (Table 4). Interventions include providing reinforcement for patients' efforts to change, providing feedback on progress, tailoring education to patients' needs and circumstances, and follow-up should be implemented.

Table 4. Summary of clinical evidence about patient-centered collaborative care and effective communication.

1. Better communication and shared decision-making improve adherence and asthma outcome

2. Health literacy is a strong predictor of a person's health, more so than age, income, employment status, education level, and race

3. Providing feedback and monitoring is a powerful behavioral strategy that improved adherence to electronic metered dose inhaler (MDI) or electronic pharmacy record

4. Interactive voice response via reminder and education calls improved adherence with steroid inhaler that was approximately one-third higher than a control group 


\section{References}

[1] Bender, B., Milgrom, H. and Rand, C. (1997) Nonadherence in Asthmatic Patients: Is There a Solution to the Problem? Annals of Allergy, Asthma \& Immunology, 79, 177-185; quiz 185-186. https://doi.org/10.1016/S1081-1206(10)63001-3

[2] Haahtela, T. (2002) The Disease Management Approach to Controlling Asthma. Respiratory Medicine, 96, S1-S8. https://doi.org/10.1016/S0954-6111(02)90076-4

[3] Milgrom, H., et al. (1996) Noncompliance and Treatment Failure in Children with Asthma. Journal of Allergy and Clinical Immunology, 98, 1051-1057. https://doi.org/10.1016/S0091-6749(96)80190-4

[4] Bender, B., et al. (1998) Psychological Factors Associated with Medication Nonadherence in Asthmatic Children. Journal of Asthma, 35, 347-353.

https://doi.org/10.3109/02770909809075667

[5] Bender, B., et al. (2000) Measurement of Children's Asthma Medication Adherence by Self Report, Mother Report, Canister Weight, and Doser CT. Annals of Allergy, Asthma \& Immunology, 85, 416-421. https://doi.org/10.1016/S1081-1206(10)62557-4

[6] Dinwiddie, R. and Muller, W.G. (2002) Adolescent Treatment Compliance in Asthma. Journal of the Royal Society of Medicine. 9, 68-71. https://doi.org/10.1177/014107680209500204

[7] Rabe, K.F., et al. (2004) Worldwide Severity and Control of Asthma in Children and Adults: The Global Asthma Insights and Reality Surveys. Journal of Allergy and Clinical Immunology, 114, 40-47. https://doi.org/10.1016/j.jaci.2004.04.042

[8] Desai, M. and Oppenheimer, J.J. (2011) Medication Adherence in the Asthmatic Child and Adolescent. Current Allergy and Asthma Reports, 11, 454-464.

https://doi.org/10.1007/s11882-011-0227-2

[9] Scarfi, C.A., et al. (2009) Association between Skin Testing in the Pediatric Emergency Department and Adherence to Follow-Up in Children with Asthma. Annals of Allergy, Asthma \& Immunology, 102, 35-40. https://doi.org/10.1016/S1081-1206(10)60105-6

[10] Mann, M., et al. (1992) A Comparison of the Effects of bid and qid Dosing on Compliance with Inhaled Flunisolide. Chest, 101, 496-499. https://doi.org/10.1378/chest.101.2.496

[11] Stoloff, S.W., et al. (2004) Improved Refill Persistence with Fluticasone Propionate and Salmeterol in a Single Inhaler Compared with Other Controller Therapies. Journal of Allergy and Clinical Immunology, 113, 245-251. https://doi.org/10.1016/j.jaci.2003.10.011

[12] Coutts, J.A., Gibson, N.A. and Paton, J.Y. (1992) Measuring Compliance with Inhaled Medication in Asthma. Archives of Disease in Childhood, 67, 332-333. https://doi.org/10.1136/adc.67.3.332

[13] Jones, C., et al. (2003) Adherence to Prescribed Treatment for Asthma: Evidence from Pharmacy Benefits Data. Journal of Asthma, 40, 93-101. https://doi.org/10.1081/JAS-120017212

[14] McNally, K.A., et al. (2009) Adherence to Combined Montelukast and Fluticasone Treatment in Economically Disadvantaged African American Youth with Asthma. Journal of Asthma. 46, 921-927. https://doi.org/10.3109/02770900903229651

[15] Fitzpatrick, A.M., et al. (2009) Tablet and Inhaled Controller Medication Refill Frequencies in Children with Asthma. Journal of Pediatric Nursing, 24, 81-89. https://doi.org/10.1016/j.pedn.2008.02.027 
[16] Carter, E.R. and Ananthakrishnan, M. (2003) Adherence to Montelukast versus Inhaled Corticosteroids in Children with Asthma. Pediatric Pulmonology, 36, 301-304. https://doi.org/10.1002/ppul.10318

[17] Lehrer, P., et al. (2002) Psychological Aspects of Asthma. Journal of Consulting and Clinical Psychology, 70, 691-711. https://doi.org/10.1037/0022-006X.70.3.691

[18] Weinstein, A.G. and Faust, D. (1997) Maintaining Theophylline Compliance/Adherence in Severely Asthmatic Children: The Role of Psychologic Functioning of the Child and Family. Annals of Allergy, Asthma \& Immunology, 79, 311-318. https://doi.org/10.1016/S1081-1206(10)63020-7

[19] Rand, C.S., et al. (1995) Long-Term Metered-Dose Inhaler Adherence in a Clinical Trial. The Lung Health Study Research Group. American Journal of Respiratory and Critical Care Medicine, 152, 580-588.

https://doi.org/10.1164/ajrccm.152.2.7633711

[20] Schmaling, K.B., McKnight, P.E. and Afari, N. (2002) A Prospective Study of the Relationship of Mood and Stress to Pulmonary Function among Patients with Asthma. Journal of Asthma, 39, 501-510. https://doi.org/10.1081/JAS-120004916

[21] Wilson, S.R., et al. (2010) Shared Treatment Decision Making Improves Adherence and Outcomes in Poorly Controlled Asthma. American Journal of Respiratory and Critical Care Medicine, 181, 566-577. https://doi.org/10.1164/rccm.200906-0907OC

[22] Borrelli, B., et al. (2007) Brief Motivational Interviewing as a Clinical Strategy to Promote Asthma Medication Adherence. Journal of Allergy and Clinical Immunology, 120, 1023-1030. https://doi.org/10.1016/j.jaci.2007.08.017

[23] Ha, J.F. and Longnecker, N. (2010) Doctor-Patient Communication: A Review. The Ochsner Journal, 10, 38-43.

[24] Rosas-Salazar, C., et al. (2012) Health Literacy and Asthma. Journal of Allergy and Clinical Immunology, 129, 935-942. https://doi.org/10.1016/j.jaci.2012.01.040

[25] Riera, A., et al. (2015) Latino Caregiver Experiences with Asthma Health Communication. Qualitative Health Research, 25, 16-26. https://doi.org/10.1177/1049732314549474

[26] Bender, G. (2016) Promoting Adherence and Effective Self-Management in Patients with Asthma. 354-359.e2.

[27] Rivera-Spoljaric, K., Halley, M. and Wilson, S.R. (2014) Shared Clinician-Patient Decision-Making about Treatment of Pediatric Asthma: What Do We Know and How Can We Use It? Current Opinion in Allergy and Clinical Immunology, 14, 161-167. https://doi.org/10.1097/ACI.0000000000000046

[28] Bukstein, D., Luskin, A.T. and Farrar, J.R. (2011) The Reality of Adherence to Rhinitis Treatment: Identifying and Overcoming the Barriers. Allergy \& Asthma Proceedings, 32, 265-271. https://doi.org/10.2500/aap.2011.32.3455

[29] Center for Health Care Strategies (2013) Fact Sheet 1: What Is Health Literacy?

[30] Eakin, M.N. and Rand, C.S. (2012) Improving Patient Adherence with Asthma Self-Management Practices: What Works? Annals of Allergy, Asthma \& Immunology, 109, 90-92. https://doi.org/10.1016/j.anai.2012.06.009

[31] Bender, B.G., et al. (2010) Test of an Interactive Voice Response Intervention to Improve Adherence to Controller Medications in Adults with Asthma. The Journal of the American Board of Family Medicine, 23, 159-165. https://doi.org/10.3122/jabfm.2010.02.090112 